\title{
A MAC Protocol With Priority Splitting Algorithm for Wireless ATM Networks
}

\author{
X. Huang and C.Tellambura \\ School of Computer Science and Software Engineering \\ Monash University \\ Clayton, Victoria 3168, Australia \\ Ph: +61 399053296 Fax: +61 399053574 \\ Email: xhuang@dgs.monash.edu.au,chintha@dgs.monash.edu.au
}

\begin{abstract}
This paper deals proposes a medium access control (MAC) protocol for ensuring the quality of service (QoS) of integrated multimedia services on wireless links. The wireless ATM MAC protocol which incorporates dynamical polling, idle uplink (UL) data channel conversion, piggybacking and an interruptable priority splitting algorithm (named THBPSA) for resolving random access collisions is proposed and named TPICC. The effect of the priority splitting algorithm on the performance of the TPICC is simulated and compared with a counterpart of the TPICC which uses an unprioritised binary splitting algorithm (UBSA) in the RA slots. The effect of an invalid polling detecting (IPD) mechanism on the UL bandwidth efficiency is also simulated. The simulation results show that the THBPSA algorithm ensures a smaller medium access delay for realtime traffic classes than for non-realtime traffic classes. Comparing THBPSA with a priority scheduling scheme which is used in the base station (BS) and features packet time stamps, THBPSA provides realtime traffic classes with a much less UL packet delay than non-realtime traffic classes. The UL bandwidth used by the dynamic polling of realtime traffic classes is tolerable.
\end{abstract}

\section{Introduction}

For the full integration of WATM mobile terminals (MTs) into fixed ATM networks, the MAC protocol is needed to transmit WATM packets through shared radio links with a duplex mode between the MTs and the BS. The duplex mode communication can either be implemented by a TDMA/FDD scheme or a TDMA/TDD scheme. Based on these schemes, a number of MAC protocols [1-7] have been proposed.

The MAC protocol should satisfy QoS requirements of each of integrated multimedia traffic classes and maximise the utilisation of the available transmission capacity. A demand assignment protocol incorporating a random access and a dynamical polling mechanism can fulfil these requirements. The design of the random access requires that the medium access delay should not only be minimised, but also should be prioritised [8-9]. Realtime traffic classes should have the minimum medium access delay.
Dynamical polling should also be used to bound the medium access delay of realtime VCs in the random access process. Without a strictly bounded medium access delay, the QoS of realtime VCs is hardly ensured. The invalid polling in a polling scheme causes the bandwidth inefficiency. The design of the protocol requires that the invalid polling should be minimised.

Thus we propose a WATM MAC protocol which incorporates both a prioritised scheme for random access and a dynamical polling scheme. We design a two-level priority splitting collision resolution algorithm, named Transcendable Hex-Binary Priority Splitting Algorithm (THBPSA), specifically for WATM traffic classes. By using THBPSA, the packet delay of realtime traffic classes is reduced and the number of invalid polling is reduced. The polling scheme is used to strictly bound the medium access delay of realtime VCs (CBR, rtVBR). By monitoring the request access (RA) slot status (collision, success and nothing), a mechanism called polling timer (PT) is used to reduce the number of invalid polling. We also include UL idle channel conversion and piggybacking [7] in the protocol to automatically provide extra bandwidth for RA requests. TPICC means THBPSA

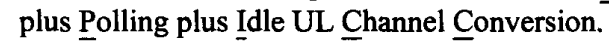

Section 2 presents the timeframe structure of the protocol. Section 3 explains the THBPSA algorithm. Section 4 describes some concepts in the design of the polling mechanism. Section 5 provides some considerations on the ICC. Section 6 describes the UL scheduling scheme. Section 7 presents some issues on the protocol operation. Section 8 reports the simulation environments and results. The conclusions are in Section 9.

\section{Protocol Timeframe Structure}

Figure 1 shows the TDMA/TDD timeframe structure which consists of a downlink (DL) subframe followed by an UL subframe. The timeframe length is fixed.

The DL subframe starts with a frame header that contains: (1) Synchronisation unique word (beacon 


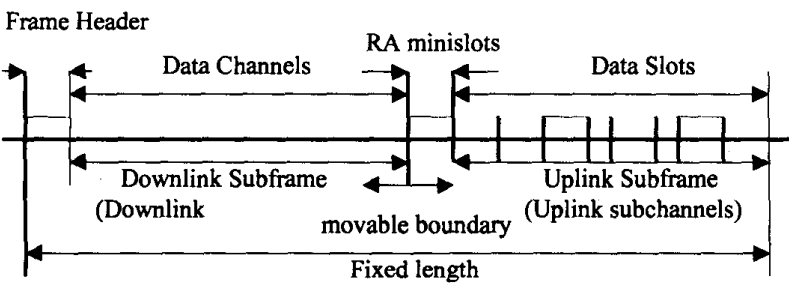

Figure 1 Wireless ATM TDMA/TDD timeframe structure

signal); (2) Acknowledgments for previous RA requests; (3) Transmission permission for each next UL data slot; (4) Channel conversion definitions (converted to temporary random access minislots or pollingresponding minislots); (5) List of polled MTs; and (6) All other necessary parts. Following the frame header are the DL data channels.

\section{THBPSA Collision Resolution Algorithm}

During a collision resolution process (CRP), we expect that the requests of realtime traffic classes are passed to the BS earlier than those of non-realtime traffic classes [8-9]. This expectation is realised by exploiting the features of the UBSA [10]. The initial splitting of the UBSA generates two groups (subsets). The requests collided in the initial collision are randomly assigned into these two groups which are marked by 1 and 2 , called group number (GN). Firstly, the request(s) in the groupl always reaches the BS ahead of the requests in the group2. The initial groupl has shorter delay. As well, during a CRP, the splitting order does not have to be identical, but can be mixed up according to practical needs. The tracing operations of a CRP are unified with the UBSA, which is: (1) When the RA feedback is "nothing" or "success", each collided MT decreases its GN by 1; (2) When the RA feedback is "collision", each collided MT increases its GN by 1 ; (3) The GN = 1 requires an MT to access the RA slot in the next timeframe; (4) The GN being 0 means the successful transmission of a $\mathrm{RA}$ request; (5) If the maximum GN generated in the initial splitting equals 0 , the current CRP finishes. This is the second feature.

Besides the original ATM traffic classes, the THBPSA takes into account the registration traffic thus resulting in a 6-level priority order: $\mathrm{CBR}>\mathrm{rtVBR}>\mathrm{nrtVBR}>$ New Registration (NR) $>$ ABR $>$ UBR. The second feature allows the THBPSA to split the initial collided MTs into six groups (splitting order $=6$ ) and then apply the UBSA (splitting order $=2$ ) to resolve each group. The splitting order is mixed up. According to the wireless ATM priority orders and the first feature, the THBPSA stipulates that, after the initial splitting, the CBR MTs, if any, choose Groupl; The rtVBR MTs, if any, choose Group2; The nrtVBR MTs, if any, choose Group3; The NR MTs, if any, choose Group4; The ABR MT, if any, choose Group5; The UBR MT, if any, choose Group6. The stipulation guarantees that a higher priority request always passes through the UL RA channel ahead of a lower one if they are in collision. This is the first level splitting operation.

The THBPSA requires that, during a CRP, new arrivals are prohibited from access the RA slots. Hence, the splitting order $=6$ in the initial splitting poses a drawback: If a CRP is very long, the possibly accumulated realtime $R A$ requests (CBR, rtVBR) will be delayed too much before the access gate opens. In order to mitigate this drawback, a second level splitting operation is designed. After the CRP of the Groupl, a probing slot (called $P_{1}$ ) is inserted to interrupt the first level splitting operations. The THBPSA requires that only the CBR requests possibly accumulated during the Group 1 CRP is allowed to access $P_{1}$. If a collision occurs in $P_{1}$, the UBSA is conducted to resolve it and then starts the Group2 CRP. At the end of the CRP of the Group2, the Group3, the Group4 and the Group5, a probing slot called $P_{2}, P_{3}, P_{4}$ and $P_{5}$ is inserted respectively. The THBPSA stipulates that the possibly accumulated $C B R$ and rtVBR requests during the first level CRP are allowed to access $P_{2} \sim P_{5}$. If a collision takes place, a priority splitting which order $=2$ is conducted. A detailed explanation and performance evaluation of the THBPSA algorithm are in [11].

\section{Polling}

\subsection{Polling Ceiling}

In the $\mathrm{BS}$, each registered realtime $\mathrm{VC}$ has a timer, called Polling Timer (PT). A Negative piggyback RA request of the VC make its $\mathrm{PT}$ start ticking from 0 based on a minimum system time unit. When the polling timer reaches a threshold called polling ceiling (PG) shown in Figure 2, the BS should poll the realtime VC in the current DL subframe and collect the answer in the following UL subframe. Setting a PG $c=\left(l_{\mathrm{d}}-1\right)$ (timeframe) results in that there will be seemingly no packets dropped due directly to the data slot access time being too long. But the packet is still possible to be dropped due to the total instantaneous bandwidth demand being too high. Polling a realtime VC with $c<$ $\left(l_{\mathrm{d}}-1\right)$ (timeframe) can statistically reduce the cell loss rate, but causes more invalid polling.

\subsection{Invalid Polling Detecting (IPD) Rules}

A set of rules allows the BS to restart (set to zero) a ticking PT before it reaching its PG, which is: (1) If all the fixed RA slots in a timeframe are not in the CRP which means that there is no realtime $R A$ request being blocked, the BS sets all ticking PT to 0. In the CRP, the THBPSA provides two more rules (2) If no collision occurs in the probing slot $P_{1}$, all CBR ticking polling timers restarts from 0 . (3) If no collision occurred in the 


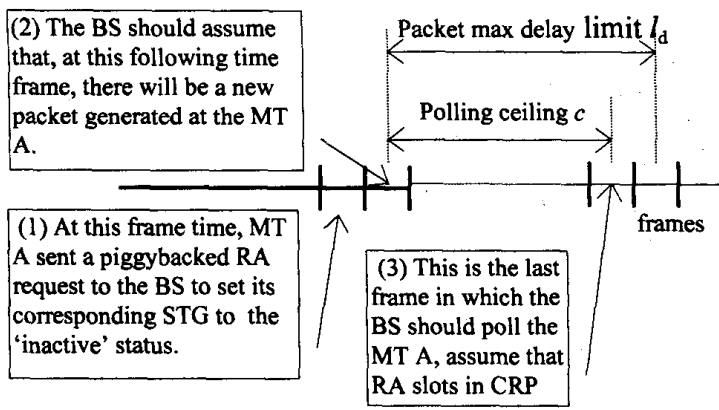

Figure 2 Polling ceiling

probing slots $\mathrm{P}_{2}, \mathrm{P}_{3}, \mathrm{P}_{4}, \mathrm{P}_{5}$, all ticking $\mathrm{CBR}$ and rtVBR PT restarts from 0.

\subsection{Dynamical Polling}

Hence, the dynamical polling for WATM can be described as: (1) Setting up a PT for each realtime VC; (2) Using negative piggyback to start the PT from 0 ; (3) Using the IPD rules to safely postpone polling actions thus reduing the number of invalid polling; (4) Flexibly adjusting the PG $c$ to statistically control the medium access delay thus to ensure the QoS.

\section{Idle UL Data Slot Conversion}

The first reason for the conversion is that the RA requests of realtime VCs needs more bandwidth (minislots) for passing the RA request to the BS within time bounds in order to fulfil QoS requirements. The second reason is the possible existence of idle UL data slots which could be exploited to meet the above bandwidth needs. The third reason is that the polling scheme also needs minislots (called polling response minislot) for MTs to respond polling.

Because the timeframe length is fixed, the design of the conversion mechanism requires an UL data slot to accommodate an integer number of minislots in order to promote the efficient use of the UL bandwidth. The

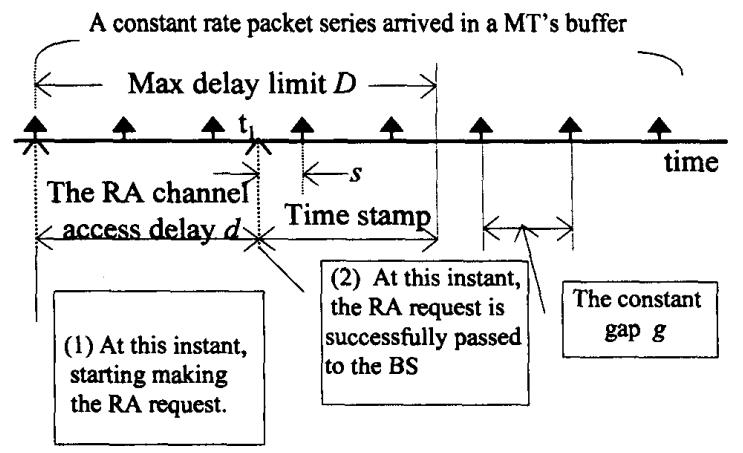

Figure 3 The time stamp is transmitted with the RA request for a CBR burst.

design of the protocol has taken this into account. In a cell with a raw bit rate of $4 \mathrm{Mbps}$ and radius $r=0.5 \mathrm{~km}$, one $1 \mathrm{~ms}$ UL subframe contains eight WATM slots with guard time of $2.33 \%$; Each slot can be converted into 9 minislots, assuming that each RA request has 5 bytes and the WATM packet has $14 \%$ overhead.

\section{UL Scheduling}

\subsection{Time Stamp}

The BS needs time stamp to optimise the scheduling of realtime traffic classes in order to reduce the cell loss rate. The time stamp is defined as a period after which a packet will be dropped. A group of instantaneous traffic parameters shown in Figure 3 must be transported to the BS for calculating the time stamps of CBR packets in an MT. At the instant $t_{1}$, the BS receives a successful $R A$ request that carries the delay $d$ of the first packet of this burst. The two parameters (1) constant gap $g$, (2) max delay limit $D$ should have been delivered to the $\mathrm{BS}$ at the initial negotiation process. Basing on these three parameters $(d, D, g)$, the BS is able to generate all the scheduling tokens for the packets that have been in the MT's buffer and calculate their time stamps. For the forthcoming packets, the BS sets a timer to the value of $s$. The timer will be decreased by 1 for each time unit. When the timer reaches zero, a scheduling token is generated and the time stamp of the token is set to the max delay limit $D$. From now on, the timer will be reset to the value of the constant gap $g$ and a scheduling token is produced every time the timer is decreased to zero. For each CBR burst, an MT only needs to transmit $d$ once.

Figure 4 shows the transmission of the rtVBR instantaneous traffic parameters. At the instant $t_{2}$, the UL data channel transmits the last packet $p_{1}$ of a MCR traffic, which carries the value of $g_{2}$ and the value of $d_{2}$ .$d_{2}$ is the delay of the first packet of the current PCR traffic. The max delay limit $D$ have been passed to the $\mathrm{BS}$ at the initial negotiation process. Basing on these three parameters $\left(d_{2}, D, g_{2}\right)$, the BS is able to generate all the scheduling tokens for the PCR packets that have been in the MT buffer and calculate their time stamps. For the forthcoming packets, the BS sets a timer to the value of $s_{2}$. The timer will be decreased by 1 for each time unit. When the timer reaches zero, a scheduling token is generated and the time stamp of the token is set to the max delay limit $D$. From now on, the timer is reset to the constant gap $g_{2}$ and a scheduling token is produced each time the timer is decreased to zero. For every rate change, the MT only needs to transmit the time stamp parameters $\left(d_{2}, g_{2}\right)$ once. In the simulation, there are two traffic rates (PCR, MCR) for rtVBR traffic classes. The extension of this scheme is also able to adapt to rtVBR traffic classes where the number of rates is larger than 2 . 


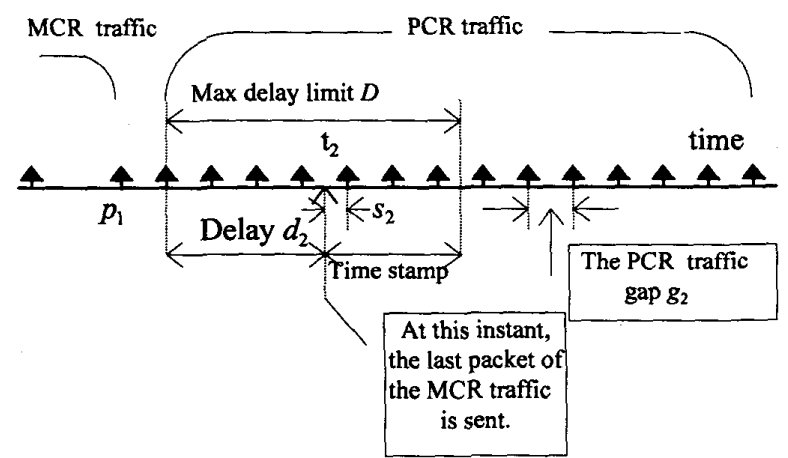

Figure 4 The time stamp is piggybacked with a data packet for variable rate bursts.

\subsection{BS UL Scheduling Strategies}

Figure 5 shows the UL scheduling mechanisms in the BS. The BS sets up a scheduling token generator (STG) for each registered UL VC. Each active STG generates a scheduling tokens (ST) for each UL packet according to the RA requests. Each ST includes an access ID; CBR and rtVBR STs also include a time stamp; The time stamp decreases itself based on a minimum system time unit. The ST will be deleted when its time stamp reaches zero. Each ST is put into a corresponding scheduling token buffer (STB). In CBR and rtVBR STB, the STs are ordered: ST with a smaller time stamp is first served. The general buffer (GB) will collect STs from those five STBs for each DL subframe. The collecting order is: 1) CBR STB; 2) rtVBR STB; 3) nrtVBR STB; 4) ABR STB; 5) UBR STB.

\section{Issues on the Protocol Operations}

For each DL, the BS first calculates the number of the MTs which are to be polled. The UL minislots for responding the polling has the first priority to use the UL bandwidth than UL data packets. After the BS finishes assigning the UL data slots and the polling minislots, the remaining UL bandwidth, if any, is allowed to be used by the converted RA minislots.

\section{Simulations, Environment and Results.}

We apply both the binary splitting algorithm [10] and the THBPSA to the RA slot of the TPICC to show the delay performance gain caused by the THBPSA. The simulation environment is shown in Table 1 and the results are in Figure 6, in which "a:a:a:a:a" represents that five ATM traffic classes has the same weight in the UL, where "a" is the number of MT. The polling and the ICC are not used and the number of the UL RA slot is one.

\section{Conclusions}

We proposed and studied a WATM MAC protocol, named TPICC. The simulation shows that: (1) The priority splitting algorithm provides a less medium access delay to realtime traffic classes. (2) The UL bandwidth used by the dynamical polling to bound the medium access delay of realtime traffic classes is small and the IPD rules can reduce the bandwidth by a limited degree. The TPICC provides a better platform for ensuring the QoS parameters.

\section{References}

[1] J. G. Kim and I. Widjaja, "PRMA/DA: A New Media Access Control Protocol for Wireless ATM." Proc. ICC'96, Dallas, TX, June 1996 pp.1-19.

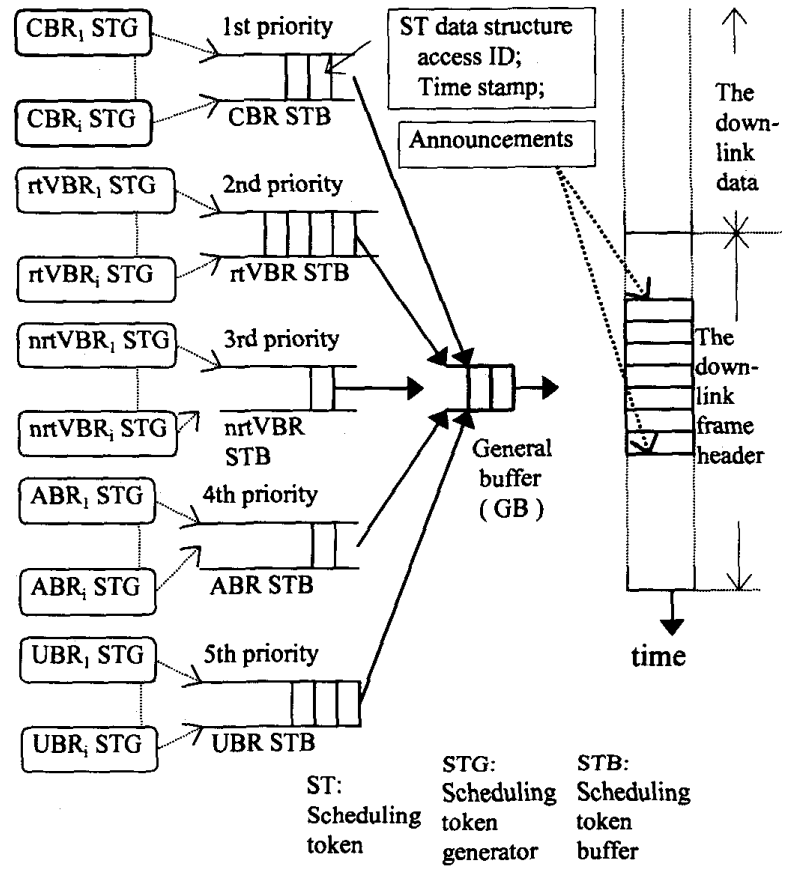

Figure 5 The scheduling mechanisms in the BS

[2] D. Petras and A. Kramling, "MAC Protocol with Polling and Fast Collision Resolution for an ATM Air Interface", IEEE ATM Wksp., San Francisco, CA, Aug. 1996.

[3] X. Qiu, V. O. K. Li and J. -H. Ju, "A Multiple Access Scheme for Multimedia Traffic in Wireless ATM", J. Special Topics in Mobile Networks and Appls. (MONET), vol. 1, no. 3, Dec. 1996, pp. 259-72. 
[4] J. G. Kim and I. Widjaja, "PRMA/DA: A New Media Access Control Protocol for Wireless ATM." Proc. ICC'96, Dallas, TX, June 1996 pp.119.

[5] D. Petras and A. Kramling, "MAC Protocol with Polling and Fast Collision Resolution for an ATM Air Interface", IEEE ATM Wksp., San Francisco, CA, Aug. 1996.

[6] X. Qiu, V. O. K. Li and J. -H. Ju, "A Multiple Access Scheme for Multimedia Traffic in Wireless ATM", J. Special Topics in Mobile Networks and Appls. (MONET), vol. 1, no. 3, Dec. 1996, pp. 259-72.

[7] F. Bauchot, S. Decrauzat, G. Marmigere, L. Merakos and N. Passas, "MASCARA, a MAC Protocol for Wireless ATM", Proc. ACTS Mobile

Table 1 Simulation Scenario

\begin{tabular}{|c|c|}
\hline Parameters & Values \\
\hline Raw bit rate & $4 \mathrm{Mbps}$ \\
\hline Cell radius & $0.5 \mathrm{~km}$ \\
\hline Wireless ATM overhead & $11 \% \sim 14 \%$ \\
\hline Error rate on wireless link & 0 (ideal case) \\
\hline Timeframe length & $2 \mathrm{~ms}$, fixed length \\
\hline DL timeframe length & $1 \mathrm{~ms}$ \\
\hline UL timeframe length & $1 \mathrm{~ms}$ \\
\hline Boundary between DL and UL & unmovable \\
\hline Number of data slot in UL & 7 \\
\hline $\begin{array}{l}\text { Number of permanent random RA } \\
\text { minislot in the UL }\end{array}$ & 1 \\
\hline $\begin{array}{l}\text { Maximum RA channel number that a } \\
\text { data channel can be converted }\end{array}$ & 9.3 \\
\hline $\begin{array}{l}\text { Maximum polling channel number } \\
\text { that a data channel can be converted }\end{array}$ & $\begin{array}{l}9 \text { (Assume that RA } \\
\text { minislot size = } \\
\text { polling-responding } \\
\text { minislot size) }\end{array}$ \\
\hline CBR PCR & $32 \mathrm{Kbps}$ \\
\hline CBR burst duration & $96 \mathrm{~ms}$ \\
\hline CBR burst interval distribution (Ave) & $\begin{array}{l}\text { Poisson (Ave }=200 \\
\text { ms) }\end{array}$ \\
\hline CBR local delay time bounds & $32 \mathrm{~ms}$ \\
\hline CBR polling ceiling & $30 \mathrm{~ms}$ \\
\hline tVBR PCR & $64 \mathrm{Kbps}$ \\
\hline rVBR MCR & $32 \mathrm{Kbps}$ \\
\hline $\begin{array}{l}\text { rVBR PCR duration } \\
\text { (occurrence probability = OP) }\end{array}$ & $48 \mathrm{~ms}(\mathrm{OP})=0.1$ \\
\hline rVBR MCR duration (probability) & $96 \mathrm{~ms}(\mathrm{OP}=0.9)$ \\
\hline $\begin{array}{l}\text { rVBR burst interval distribution } \\
\text { (Ave) }\end{array}$ & Poisson $(200 \mathrm{~ms})$ \\
\hline rVBR local delay bound $\left(l_{\mathrm{d}}\right)$ & variable in Figure 7 \\
\hline rVBR polling ceiling & $=\left(l_{\mathrm{d}}-1\right)$ timeframe \\
\hline nrtVBR, ABR, UBR & $\begin{array}{l}\text { (The same as rtVBR, } \\
\text { but no delay bounds } \\
\text { and polling ceiling) }\end{array}$ \\
\hline NR (new registration) buffer & $\begin{array}{l}5 \text { (generating RA } \\
\text { traffic, But no data } \\
\text { traffic) }\end{array}$ \\
\hline NR Ave. rate & 5 Times per second \\
\hline $\begin{array}{l}\text { NR interval distribution (Ave) } \\
\text { Summit, Nov } 1996, \mathrm{pp} \text {. }\end{array}$ & Poisson $(200 \mathrm{~ms})$ \\
\hline
\end{tabular}

[8] F. D. Priscoli, "Medium Access Control for the MEDIAN System", Proc. ACTS Mobile Summit'96, Granada, Spain, Nov. 1996, pp. 1-8.

[9] H. Xie et al., "Data Link Control Protocols for Wireless ATM Access Channels," Proc. of ICUPC'95, Tokyo, Japan, Nov. 1995, pp. 1-5.

[10]M. J. Karol, Z. Liu, K. Y.. Eng, "Distributed Queueing Request Update Multiple Access (DQRUMA) for Wireless Packet (ATM) Networks", Proc. ICC'95, June 1995, pp. 1224-31.

[11]J. Sanchez, Rmartinez and M. WMarcellin, "A Survey of MAC Protocols Proposed for Wireless ATM", IEEE Network, November/December, 1997, pp. 52 -- 62.

[12] O. Kubbar and H. T.Mouftah, "Multiple Access Control Protocols for Wireless ATM: Problems Definition and Design Objectives", IEEE Communications Magazine, Nov1997, pp. 93 99.

[13]Raphael Rom, Moshe Sidi, Multiple Access Protocols Performance and Analysis, Oct 1989.

[14]X. Huang and C.Tellambura, "A collision resolution algorithm for random multiple access supporting integrated ATM services on wireless links", IEEE International Conference on Third Generation Wireless Communications, Silicon Valley, June, USA.

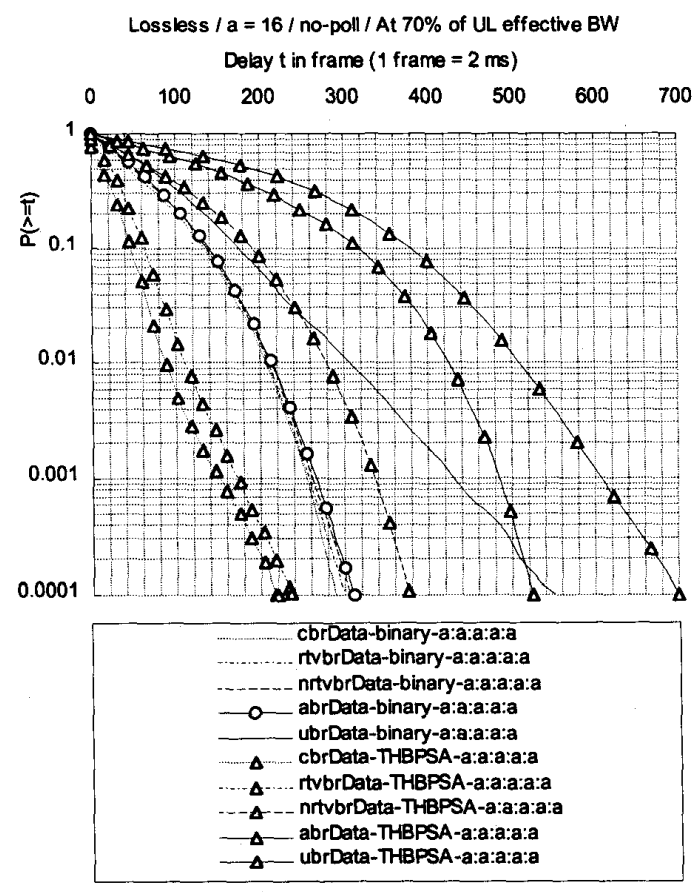

Figure 6 Delay complementary distributions 